\title{
APPROXIMATING THE PROJECTIVE
}

MODEL

\author{
Evangelos Kranakis \\ Centrum voor Wiskunde en Informatica \\ P.O. Box 4079, 1009 AB Amsterdam, The Netherlands
}

\begin{abstract}
One of the fundamental questions in the calculus of communicating processes is determining if a given system of fixed point equations has a solution in the projective model. The present paper provides an approximation principle for the projective model, which makes it posssible to prove assertions in this model by proving them in an infinite sequence of certain finite process algebras. Motivated from this principle a new model for process algebras is defined and its relationship to the projective model is studied.
\end{abstract}

Mathematics Subject Classification: 68B05.

Key Words and Phrases: process algebra, process, projective model, polynomial operator, metric space, approximation principle, positive formulas, ultrafilter, ultraproduct.

Acknowledgements: This research was carried out while the author was visiting the Computer Science Department of the University of Amsterdam and it was partially supported by Esprit under contract no. 432, Meteor. I would like to thank all the participants of the P.A.M. seminar for their numerous comments. However, I am particularly indebted to J. Baeten, R. van Glabbeek, J. W. Klop and F. Vaandrager, whose valuable criticisms helped me correct my numerous errors.

\section{Introduction}

In the formal analog of Milner's work on Calculus of Communicating Systems (see [M]), as described by Bergstra and Klop in [BK], one builts large systems of processes by assembling together atomic processes (or actions) chosen from a finite set $A$ of such atomic processes (see $[\mathrm{H}])$. These systems of processes satisfy a set of equational laws, called the axioms of the theory of the algebras of communicating processes (or theory of process algebras). The models of this theory are called process algebras. Its axioms are described in a signature that includes: + (alternative composition or sum), $\cdot($ sequential composition or product), I| (parallel composition or merge), $\mathbb{L}$ (left merge), | (communication merge), $\partial_{H}$ (encapsulation, where $H$ is a subset of the set $A$ of atoms), the atomic process $\delta$ (deadlock or failure) and the atom $a$, for each $a \in A$.

In the table below the equational laws for process algebras are given. The communication function | : $A_{\delta} \times A_{\delta} \rightarrow A_{\delta}$ (where $A_{\delta}$ consists of the atoms in $A$ including $\delta$ ) is initially defined only on atomic processes. Then it is extended to all finite terms using the merge 
and communication axioms. In the absence of communication, the axiom $x\|y=y\|$ $x+x \mathbb{L}+x \mid y$ should be replaced with the new axiom $x \| y=y \mathbb{L}+x \mathbb{L}$. The theory consisting of the first five basic axioms together with the first four communication axioms is known as (basic) process algebra and is abbreviated by $P A$. ACP, the algebra of communicating processes consists of the basic, merge, communication and encapsulation axioms. As usual, the universal quantifiers, which quantify the variables $x, y, z$ in the axioms below are omitted. In addition, the letters $a, b$ range over $A$. The axioms of process algebras are the following:

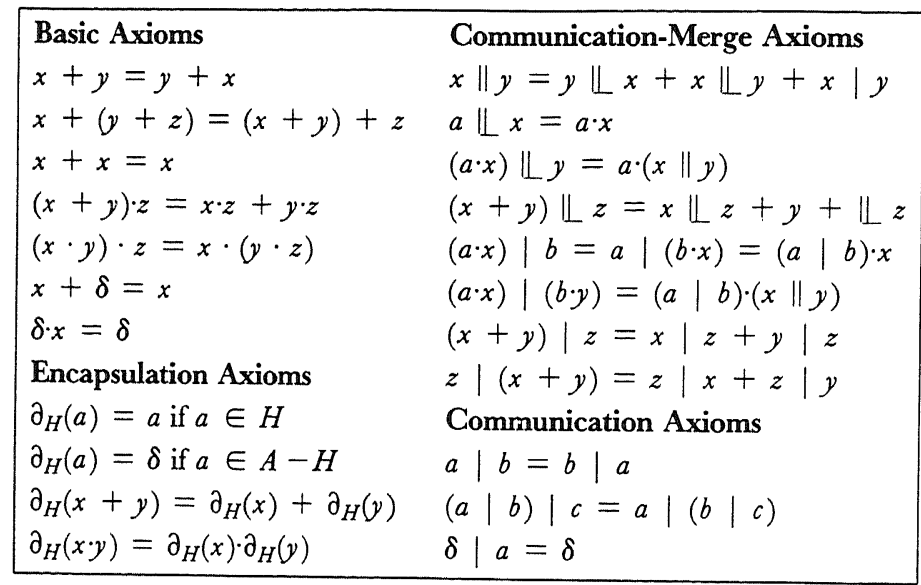

In this axiomatic framerwork one can define the so-called term (or initial) model $A_{\omega}$, i.e. the least set $S$ of finite strings such that $S$ contains all the constants of the given signature, and $S$ is closed under the operations of the given signature. (The reader should be aware of all the possible signatures arising in the present study; practically every subset of $+, \cdot, \|, \mathbb{L}, \mid, \partial_{H}, \delta, a(a \in A)$ is a possible signature and hence it can give rise to a different term model $A_{\omega}$. It would be very cumbersome however to keep a different notation for $A_{\omega}$ for each possible signature. Instead, it will be left to the reader to derive from the context what the proper signature in each case is.)

Given any term $t$ in $A_{\omega}$ and any positive integer $n$ let $(t)_{n}$ be the subtree of $t$ of height at most $n$ obtained from $t$ by deleting all those nodes which are located at height bigger than n. More formally define:

$$
\begin{aligned}
& (a)_{n}=a, \\
& (a t)_{1}=a, \\
& (a t)_{n}=a(t)_{n-1}, \text { for } n>1, \\
& \left(t+t^{\prime}\right)_{n}=(t)_{n}+\left(t^{\prime}\right)_{n}, \text { for } n>0 .
\end{aligned}
$$

Now $A_{n}$ is defined to be the set $\left\{(t)_{n}: t \in A_{\omega}\right\}$. In a sense, $(\cdot)_{n}$ can be considered as the projection of the term model $A_{\omega}$ onto the model $A_{n}$. For each binary operation $\square$ on $A_{\omega}$ define an operation $\square_{n}$ on $A_{n}$ by $t \square_{n} s=(t \square s)_{n}$ (the case of the operation $\partial_{H}$ is treated similarly). This makes each $A_{n}$ into a process algebra. The projective (or standard) model, denoted by $A^{\infty}$ consists of all infinite sequences $\left(p_{1}, p_{2}, \ldots, p_{n}, \ldots\right)$ such that $p_{n} \in A_{n}$ and $\left(p_{n+1}\right)_{n}=p_{n}$, for all $n>0$. The operations are defined on $A^{\infty}$ in a natural way; thus, following $[\mathrm{BK}]$, if $\square$ is any binary operation on $A_{\omega}$ one defines a new binary opera- 
tion $\square^{\prime}$, which for convenience will also be denoted by $\square$, as follows:

$$
\left(p_{1}, \ldots, p_{n}, \ldots\right) \square\left(q_{1}, \ldots, q_{n}, \ldots\right)=\left(\left(p_{1} \square q_{1}\right)_{1}, \ldots,\left(p_{n} \square q_{n}\right)_{n}, \ldots\right)
$$

Remark on Notation: Throughout the present paper $T\left(x_{1}, \ldots, x_{n}\right), S\left(x_{1}, \ldots, x_{n}\right)$, etc. with or without subscripts and superscripts, will always denote (polynomial) operators, i.e. terms built up from the atomic processes, the variables $x_{1}, \ldots, x_{n}$, the atoms in $A$ and the operations of the given signature.

The class $P$ of positive formulas is the smallest class of well founded formulas in the signature $+, \cdot, \|, \mathbb{L}, \mid, \partial_{H}, \delta, a(a \in A)$, which satisfies the following properties:

(a) For all polynomial operators $T, S, T\left(v_{1}, \ldots, v_{n}\right)=S\left(v_{1}, \ldots, v_{n}\right)$ is in $P$.

(b) If $\Phi, \Psi \in P$ then $\Phi \vee \Psi \in P$.

(c) For any countable $\Delta \subseteq P$, the formula $\wedge \Delta$ is in $P$.

(d) If $\Phi\left(v_{1}, \ldots, v_{n}, \ldots\right) \in P$ and the variables $u_{1}, \ldots, u_{k}, \ldots$ are from the set $\left\{v_{1}, \ldots, v_{n}, \ldots\right\}$ then both formulas $\left(\exists u_{1} \cdots \exists u_{k} \cdots\right) \Phi,\left(\forall u_{1} \cdots \forall u_{k} \cdots\right) \Phi$ are in $P$.

The class $P_{0}$ of finite positive formulas is the smallest class of well founded formulas in the signature $+, \cdot \|, \mathbb{L}, \mid, \partial_{H}, \delta, a(a \in A)$, which satisfies the following properties:

(a) For all polynomial operators $T, S, T\left(v_{1}, \ldots, v_{n}\right)=S\left(v_{1}, \ldots, v_{n}\right)$ is in $P$.

(b) If $\Phi, \Psi \in P$ then $\Phi \vee \Psi, \Phi \wedge \Psi \in P$.

(c) If $\Phi\left(v_{1}, \ldots, v_{n}\right) \in P$ and $u_{1}, \ldots, u_{k} \in\left\{v_{1}, \ldots, v_{n}\right\}$ then both formulas $\left(\exists u_{1} \cdots \exists u_{k}\right) \Phi$, $\left(\forall u_{1} \cdots \forall u_{k}\right) \Phi$ are in $P$

Most of section 3 will be dedicated to a proof of the following theorem.

Theorem 1.1. [Approximation Theorem] Any formula $\Phi\left(v_{1}, \ldots, v_{n}, \ldots\right) \in P$ satisfies the following approximation principle: for any convergent sequences $\left\{x_{1, n}\right\}, \ldots,\left\{x_{k, n}\right\}, \ldots$ such that $x_{k, n} \in A_{n}$ for all $k, n$, if the set $\left\{n>0: A_{n} \models \Phi\left(x_{1, n}, \ldots, x_{k, n}, \ldots\right)\right\}$ is infinite then it is true that $A^{\infty}=\Phi\left(\lim _{n \rightarrow \infty} x_{1, n}, \ldots, \lim _{n \rightarrow \infty} x_{k, n}, \ldots\right)$.

Such formulas $\Phi$ occur when one wants to prove that a system of fixed point equations has a solution. As an example, consider the infinite system

$$
\Sigma=\left\{x_{k}=T_{k}\left(x_{1}, \ldots, x_{n(k)}\right): k>0\right\},
$$

where each $T_{k}$ is a polynomial operator in the indicated variables. The assertion $\Sigma$ has a solution in $A^{\infty}$ can be expressed by the formula: $\left(\exists x_{1} \cdots \exists x_{k} \cdots\right) \Psi$, where $\Psi$ is the countable conjunction of all the formulas $x_{k}=T_{k}\left(x_{1}, \ldots, x_{n(k)}\right)$, for $k>0$. Theorem 1.1 states that in order to prove that $\Sigma$ has a solution in $A^{\infty}$, it is enough to show that $\Sigma$ has a solution in infinitely many $A_{n}$ 's. For more specific examples of systems the reader is referred to $[\mathrm{BK}],[\mathrm{H}]$ and $[\mathrm{K}]$.

It is also possible to prove a partial converse of the approximation principle. 
Theorem 1.2. [Converse of the Approximation Principle] For any formula $\Phi\left(v_{1}, \ldots, v_{k}, \ldots\right) \in P$ and any $p_{1}, \ldots, p_{k}, \ldots \in A^{\infty}$ the following statements are equivalent:

(i) $A^{\infty}=\Phi\left(p_{1}, \ldots, p_{k}, \ldots\right)$.

(ii) $\left\{n>0: A_{n} \models \Phi\left(\left(p_{1}\right)_{n}, \ldots,\left(p_{k}\right)_{n}, \ldots\right)\right\}$ is infinite.

(iii) $\forall n>0\left[A_{n} \models \Phi\left(\left(p_{1}\right)_{n}, \ldots,\left(p_{k}\right)_{n}, \ldots\right)\right]$.

Motivated from the approximation theorem one can define a new process algebra, which is an extension of the projective algebra $A^{\infty}$. To state the next theorem the notion of ultrafilter on the set $N$ of positive integers will be required. Call $D$ a (nonprincipal) ultrafilter on $N$ if $D$ is a nonempty set of subsets of $N$ satisfying the following properties for all $X, Y \subseteq N$ : (i) $\varnothing$ is not a member of $D$, (ii) if $X \subseteq Y$ and $X \in D$ then $Y \in D$, (iii) $X \in D$ or $N-X \in D$, (iv) if $X, Y \in D$ then $X \cap Y \in D$ and (v) if $X \in D$ then $X$ is infinite. Notice that the existence of such ultrafilters requires the axiom of choice (see $[E]$ or [CK]).

The main theorem of section 4 is the following.

Theorem 1.3. For any ultrafilter $D$ on the set $N$ of positive integers there exists a process algebra $A^{D}$, which is a proper extension of the projective algebra $A^{\infty}$. Moreover, for any finite, positive formula $\Phi\left(v_{1}, \ldots, v_{k}\right) \in P_{0}$ and any $p_{1}, \ldots, p_{k} \in A^{\infty}$ the following statements are equivalent:

(i) $A^{D}=\Phi\left(p_{1}, \ldots, p_{k}\right)$.

(ii) $A^{\infty}=\Phi\left(p_{1}, \ldots, p_{k}\right)$.

(iii) $\left\{n>0: A_{n} \models \Phi\left(\left(p_{1}\right)_{n}, \ldots,\left(p_{k}\right)_{n}\right)\right\} \in D$.

(iv) $\left\{n>0: A_{n}=\Phi\left(\left(p_{1}\right)_{n}, \ldots,\left(p_{k}\right)_{n}\right)\right\}$ is infinite.

(v) $\forall n>0\left[A_{n} \models \Phi\left(\left(p_{1}\right)_{n}, \ldots,\left(p_{k}\right)_{n}\right)\right]$

\section{Topology of the Projective Model}

As explained above the projective model $A^{\infty}$ consists of all sequences $\left(p_{1}, \ldots, p_{n}, \ldots\right)$ such that each $p_{n} \in A_{n}$ and $\left(f_{n+1}\right)_{n}=p_{n}$, for all $n>0$. The term model $A_{\omega}$ can be embedded in a natural way in the projective model $A^{\infty}$; for any finite term $t$ associate the infinite sequence $p(t)=\left((t)_{1}, \ldots,(t)_{n}, \ldots\right)$. Because of this it is identified with a subset of the projective model (this also explains why the same symbol is used for the corresponding operations in $\left.A_{\omega}, A^{\infty}\right)$. Extend the projection functions to $A^{\infty}$ by defining $(p)_{n}=p_{n}$, for all $p=\left(p_{1}, \ldots, p_{n}, \ldots\right) \in A^{\infty}$ and all $n>0$. For any two distinct elements $p, q$ of $A^{\infty}$ let $k(p, q)=$ the least $n>0$ such that $(p)_{n}$ is not equal to $(q)_{n}$. This definition makes it possible to endow $A^{\infty}$ with a metric space structure. Indeed, define the distance $d(p, q)$ between $p, q$ by

$$
d(p, q)= \begin{cases}2^{-k(p, q)} & \text { if } p \neq q \\ 0 & \text { if } p=q .\end{cases}
$$


This metric was used by Arnold and Nivat (see [AN]) in the context of Denotational Semantics of Concurrency. An essentially equivalent metric was also defined by de Bakker and Zucker (see $[\mathrm{dBZ}]$ ). For additional information and further properties of this metric the reader is advised to consult $[\mathrm{L}]$ and $[\mathrm{Ro}]$.

The following results summarize all the basic properties of the metric space $\left(A^{\infty}, d\right)$ and will be used frequently in the sequel. Their proof is omitted, but the interested reader can easily derive the essential details from $[\mathrm{Du}],[\mathrm{L}],[\mathrm{AN}]$ and $[\mathrm{K}]$.

Theorem 2.1. [In the signature $+, \cdot, \|, \mathbb{L}, \mid, \partial_{H}, \delta, a(a \in A)$ ]

(i) $\left(A^{\infty}, d\right)$ is an ultrametric space, i.e. it satisfies the following three properties for all elements $p, q, r \in A^{\infty}$,

$$
\begin{aligned}
& \text { (a) } d(p, q)=0 \text { if and only if } p=q, \\
& \text { (b) } d(p, q)=d(q, p), \\
& \text { (c) } d(p, q) \leqslant \max \{d(p, r), d(r, q)\} .
\end{aligned}
$$

(ii) $p^{(r)} \rightarrow p$ if and only if $\forall n \exists m \forall k \geqslant m\left[\left(p^{(k)}\right)_{n}=(p)_{n}\right]$.

(iii) $\left(A^{\infty}, d\right)$ is the metric completion of the metric space $\left(A_{\omega}, d^{\prime}\right)$, where $d^{\prime \prime}$ is the restriction of $d$ on $A_{\omega}$.

(iv) For all $p \in A^{\infty}$ and each $n>0, d\left(p,(p)_{n}\right) \leqslant 2^{-n}$. Hence, $\lim _{n \rightarrow \infty}(p)_{n}=p$.

(v) The operations $(\cdot)_{n}: A^{\infty} \rightarrow A_{n}$ are continuous.

(vi)Any operator $T\left(x_{1}, \ldots, x_{n}\right)$ is continuous in the variables $x_{1}, \ldots, x_{n}$. In fact, for all $p_{1}, \ldots, p_{n}, q_{1}, \ldots, q_{n} \in A^{\infty}$

$$
d\left(T\left(p_{1}, \ldots, p_{n}\right), T\left(q_{1}, \ldots, q_{n}\right)\right) \leqslant \max \left\{d\left(p_{1}, q_{1}\right), \ldots, d\left(p_{n}, q_{n}\right)\right\} .
$$

(vii) $A$ is finite if and only if $\left(A^{\infty}, d\right)$ is compact.

In view of this last theorem from now on and for the rest of the paper it will be always assumed that $A$ is finite. This will guarantee that $A^{\infty}$ is compact.

\section{The Approximation Principle}

Intuitively, the approximation principle enables one to verify assertions in the projective model by proving that the same assertion is valid in infinitely many $A_{n}$ 's. To be more specific, a formula $\Phi$ is said to satisfy the approximation principle, and this will be abbreviated by $A(\Phi)$, if the following property holds: for any convergent sequences $\left\{x_{1, n}\right\}, \ldots,\left\{x_{k, n}\right\}, \ldots$ such that $x_{k, n} \in A_{n}$ for all $k, n$, if the set

$$
\left\{n>0: A_{n} \models \Phi\left(x_{1, n}, \ldots, x_{k, n}, \ldots\right)\right\}
$$

is infinite then it is true that

$$
A^{\infty}=\varphi\left(\lim _{n \rightarrow \infty} x_{1, n}, \ldots, \lim _{n \rightarrow \infty} x_{k, n}, \ldots\right) .
$$

Now it is possible to prove theorem 1.1. 
Proof of theorem 1.1: It is enough to show that each formula $\Phi \in P$ satisfies $A(\Phi)$. The proof is by induction on the construction of $\Phi$.

Case 1: $\Phi \equiv T\left(v_{1}, \ldots, v_{m}\right)=S\left(v_{1}, \ldots, v_{m}\right)$, where $T, S$ are polynomial operators.

For any operator $T\left(v_{1}, \ldots, v_{m}\right)$ let $T^{n}\left(v_{1}, \ldots, v_{m}\right)$ denote the interpretation of $T$ in the model $A_{n}$. Using induction on the construction of $T$ and the definitions of the operations in the process algebras $A_{n}$ (see section 1 ) it is easy to show that

Lemma 3.1. For all $x_{1}, \ldots, x_{m} \in A_{n}, T^{n}\left(x_{1}, \ldots, x_{m}\right)=\left(T\left(x_{1}, \ldots, x_{m}\right)\right)_{n}$.

Now it is required to show that the formula $T\left(v_{1}, \ldots, v_{m}\right)=S\left(v_{1}, \ldots, v_{m}\right)$ satisfies the above approximation principle. Indeed, let $\left\{x_{1, n}\right\}, \ldots,\left\{x_{k, n}\right\}, \ldots$ be any convergent sequence such that $x_{k, n} \in A_{n}$ for all $k, n$ and the set

$$
J=\left\{n>0: A_{n} \models T\left(x_{1, n}, \ldots, x_{m, n}\right)=S\left(x_{1, n}, \ldots, x_{m, n}\right)\right\}
$$

is infinite. It is enough to show that

$$
A^{\infty}=T\left(\lim _{n \rightarrow \infty} x_{1, n}, \ldots, \lim _{n \rightarrow \infty} x_{m, n}\right)=S\left(\lim _{n \rightarrow \infty} x_{1, n}, \ldots, \lim _{n \rightarrow \infty} x_{m, n}\right) .
$$

Put $\left(x_{1}, \ldots, x_{m}\right)=\left(\lim _{n \rightarrow \infty} x_{1, n}, \ldots, \lim _{n \rightarrow \infty} x_{m, n}\right)$. It is clear that for all $n \in J$,

$$
T^{n}\left(x_{1, n}, \ldots, x_{m, n}\right)=S^{n}\left(x_{1, n}, \ldots, x_{m, n}\right) .
$$

Using this last equation and lemma 3.1 it is clear that for all $n \in J$,

$$
\left(T\left(x_{1, n}, \ldots, x_{m, n}\right)\right)_{n}=\left(S\left(x_{1, n}, \ldots, x_{m, n}\right)\right)_{n} .
$$

The following result is an easy consequence of the definition of convergence in $A^{\infty}$ :

Lemma 3.2. For any sequence $\left\{u_{n}\right\}$ of terms in $A_{\omega}$, if $u_{n} \rightarrow u$ then $\left(u_{n}\right)_{n} \rightarrow u$.

Using the continuity of the operators $T, S$ (see theorem 2.1) it follows that

$$
T^{n}\left(x_{1, n}, \ldots, x_{m, n}\right) \rightarrow T\left(x_{1}, \ldots, x_{m}\right), S^{n}\left(x_{1, n}, \ldots, x_{m, n}\right) \rightarrow S\left(x_{1}, \ldots, x_{m}\right) .
$$

Hence, using lemma 3.2 as well as (1) and (2) it follows that

$$
A^{\infty}=T\left(x_{1}, \ldots, x_{m}\right)=S\left(x_{1}, \ldots, x_{m}\right),
$$

which completes the proof in case 1 .

Case 2: $\Phi \equiv \Theta \vee \Psi$.

Let $\left\{x_{1, n}\right\}, \ldots,\left\{x_{k, n}\right\}, \ldots$ be a convergent sequence such that $x_{k, n} \in A_{n}$ for all $k, n$, and the set $J=\left\{n>0: A_{n} \models \Phi\left(x_{1, n}, \ldots, x_{k, n}, \ldots\right)\right\}$ is infinite. To show that

$$
A^{\infty}=\Phi\left(\lim _{n \rightarrow \infty} x_{1, n}, \ldots, \lim _{n \rightarrow \infty} x_{k, n}, \ldots\right) \text {. }
$$

Put $\quad K=\left\{n>0: A_{n} \models \Theta\left(x_{1, n}, \ldots, x_{k, n}, \ldots\right)\right\}, \quad L=\left\{n>0: A_{n} \models \Psi\left(x_{1, n}, \ldots, x_{k, n}, \ldots\right)\right\}$. Since 
$J=K \cup L$ it is clear that at least one of the sets $K, L$ (say $K$ ) is infinite. It follows from the induction hypothesis that

$$
A^{\infty}=\Theta\left(\lim _{n \rightarrow \infty} x_{1, n}, \ldots, \lim _{n \rightarrow \infty} x_{k, n}, \ldots\right)
$$

and hence also

$$
A^{\infty}=\Phi\left(\lim _{n \rightarrow \infty} x_{1, n}, \ldots, \lim _{n \rightarrow \infty} x_{k, n}, \ldots\right)
$$

which completes the proof of case 2 .

Case 3: $\Phi \equiv \bigwedge\left\{\Phi_{i}: i>0\right\}$.

Let $\left\{x_{1, n}\right\}, \ldots,\left\{x_{k, n}\right\}, \ldots$ be a convergent sequence such that $x_{k, n} \in A_{n}$ for all $k, n$, and the set $J=\left\{n>0: A_{n} \models \Phi\left(x_{1, n}, \ldots, x_{k, n}, \ldots\right)\right\}$ is infinite. To show that

$$
A^{\infty}=\Phi\left(\lim _{n \rightarrow \infty} x_{1, n}, \ldots, \lim _{n \rightarrow \infty} x_{k, n}, \ldots\right) \text {. }
$$

For each $i>0$ put $J_{i}=\left\{n>0: A_{n} \models \Phi_{i}\left(x_{1, n}, \ldots, x_{k, n}, \ldots\right)\right\}$. Clearly, each $J_{i}$ is infinite and hence the induction hypothesis implies that for all $i>0$,

$$
A^{\infty}=\Phi_{i}\left(\lim _{n \rightarrow \infty} x_{1, n}, \ldots, \lim _{n \rightarrow \infty} x_{k, n}, \ldots\right)
$$

which completes the proof of case 3 .

Case 4: $\Phi \equiv\left(\exists u_{1} \cdots \exists u_{k} \ldots\right) \Psi\left(u_{1}, \ldots, u_{k}, \ldots, v_{1}, \ldots, v_{n}, \ldots\right)$

Actually this is the only part of the proof which requires the compactness of $A^{\infty}$. Let $\left\{x_{1, n}\right\}, \ldots,\left\{x_{k, n}\right\}, \ldots$ be a convergent sequence such that $x_{k, n} \in A_{n}$ for all $k, n$, and the set $J=\left\{n>0: A_{n} \models \Phi\left(x_{1, n}, \ldots, x_{k, n}, \ldots\right)\right\}$ is infinite. To show that

$$
A^{\infty}=\Phi\left(\lim _{n \rightarrow \infty} x_{1, n}, \ldots, \lim _{n \rightarrow \infty} x_{k, n}, \ldots\right) \text {. }
$$

By assumption, for each $n \in J$ there exist elements $y_{k, n} \in A_{n}$ such that

$$
A_{n} \models \Psi\left(y_{1, n}, \ldots, y_{k, n}, \ldots, x_{1, n}, \ldots, x_{k, n}, \ldots\right) \text {. }
$$

The sequences $\left(\left\{y_{k, n}\right\}: k>0\right)$ need not be convergent. However, using the compactness of the metric space $A^{\infty}$ (in fact one needs the compactness of the cartesian product space of countable many copies of $A^{\infty}$ ) there exists an infinite subset $L$ of $J$ such that each of the sequences $\left\{y_{k, n}\right\}_{n} \in L$ is convergent. For each $k$ let $y_{k}$ be the limit of this last sequence. Now apply the induction hypothesis to the formula $\Psi$ and the convergent sequences $\left\{x_{k, n}\right\}_{n} \in L$ $\left\{y_{k, n}\right\}_{n} \in L$, for $k>0$, to obtain that

$$
A^{\infty}=\Psi\left(y_{1}, \ldots, y_{k}, \ldots, x_{1}, \ldots, x_{k}, \ldots\right)
$$

This completes the proof of case 4 .

Case 5: $\Phi \equiv\left(\forall u_{1} \cdots \forall u_{k} \cdots\right) \Psi\left(u_{1}, \ldots, u_{k}, \ldots, v_{1}, \ldots, v_{n}, \ldots\right)$

This is similar to the cases above and its proof is left to the reader. Now the proof of theorem 1.1 is complete. 
The examples given below indicate that theorem 1.1 is best possible, in the sense that the approximation principle is not invariant neither under infinitary disjunctions nor under negations.

Example 3.3. [Noninvariance under infinitary disjunctions] Consider the formula $\Phi \equiv \bigvee\left\{x=a^{n}: n>0\right\}$. For each $n>0 A_{n} \models \Phi\left(a^{n}\right)$; however, $A^{\infty} \models \Phi\left(a^{\omega}\right)\left(a^{\omega}\right.$ is the limit of the sequence $a^{n}$ in $A^{\infty}$ ). An even better example involving a sentence (due to $\mathrm{H}$. Mulder $)$ is $\Phi \equiv \bigvee\left\{\exists x\left(x a=a^{n}\right): n>0\right\}$.

Example 3.4. [Noninvariance under negations] Consider the formula $\Psi(x, y) \equiv x \neq y$. Clearly, for all $n>0 A_{n} \models \Psi\left(a^{n}, a^{n-1}\right)$; however, $A^{\infty} \models \neg \Psi\left(a^{\omega}, a^{\omega}\right)$.

Proof of theorem 1.2: In view of theorem 1.1 it is enough to prove that (i) implies (iii). In fact, it is enough to show by induction on positive formulas $\Phi$ that for all $p_{1}, \ldots, p_{k}, \ldots \in A^{\infty}$,

$$
A^{\infty}=\Phi\left(p_{1}, \ldots, p_{k}, \ldots\right) \Rightarrow \forall n>0\left[A_{n} \models \Phi\left(\left(p_{1}\right)_{n}, \ldots,\left(p_{k}\right)_{n}, \ldots\right)\right]
$$

The initial step of the proof is for formulas of the form $T\left(v_{1}, \ldots, v_{k}\right)=S\left(v_{1}, \ldots, v_{k}\right)$. Suppose that $p_{1}, \ldots, p_{k} \in A^{\infty}$ such that $A^{\infty}=T\left(p_{1}, \ldots, p_{k}\right)=S\left(p_{1}, \ldots, p_{k}\right)$. Then for all $n>0$,

$$
\left(T^{n}\left(\left(p_{1}\right)_{n}, \ldots,\left(p_{k}\right)_{n}\right)_{n}=\left(T\left(p_{1}, \ldots, p_{k}\right)\right)_{n}=\left(S\left(p_{1}, \ldots, p_{k}\right)\right)_{n}=\left(S^{n}\left(\left(p_{1}\right)_{n}, \ldots,\left(p_{k}\right)_{n}\right)_{n},\right.\right.
$$

as desired. The rest of the proof is much like the proof of theorem 1.1 and is left to the reader.

\section{The Ultraproduct Model}

The most natural way to interpret the approximation principle is via the ultraproduct model. Details of its definition and fundamental properties can be found in [CK] and [E]. Given an ultrafilter $D$ on $N$, define the equivalence relation $\equiv_{D}$ on the product set $\Pi\left\{A_{n}: n>0\right\}$, as follows: $f \equiv_{D} g$ if and only if $\{n>0: f(n)=g(n)\} \in D$. Call $[f]_{D}$ the equivalence class of $f$ modulo $\equiv_{D}$ and let $A^{D}$ be the set of these equivalence classes. $A^{D}$ can be turned into a process algebra by defining an operation $\square_{D}$, for any binary operation $\square$, as follows:

$$
[f]_{D} \square_{D}[g]_{D}=\left[f(n) \square_{n} g(n): n>0\right]_{D} .
$$

(The unary operation $\partial_{H}$ is treated similarly.) It turns out that the mapping $p \rightarrow\left[(p)_{n}: n>0\right]_{D}$ is a homomorphic embedding of $A^{\infty}$ into $A^{D}$. This makes it possible to identify the elements of $A^{\infty}$ with their corresponding images in $A^{D}$ via the above embedding, and hence consider $A^{\infty}$ as a subset of $A^{D}$.

Proof of theorem i.3: Let $\Phi\left(v_{1}, \ldots, v_{k}\right)$ be a finite positive formula in the given signature. The equivalence of (i), (iii) is a consequence of the fundamental theorem for ultraproducts (see [E]). Since the ultrafilter $D$ is nonprincipal (i.e. all its elements are infinite sets) the implications $(\mathrm{v}) \Rightarrow$ (iii) $\Rightarrow$ (iv) are also immediate. The implication (iv) $\Rightarrow$ (ii) is a consequence of the approximation theorem and the fact that $\lim _{n \rightarrow \infty}(p)_{n}=p$. Finally the implication (ii) $\Rightarrow(v)$ is a special case of theorem 1.2. 
Example 4.1. Theorem 1.3 cannot be extended to a set of formulas which is closed under negation. To see this consider the formula $\phi \equiv(\exists x, y)\left[x=a x \wedge y^{2}=a y^{2} \wedge x \neq y\right]$. The fundamental theorem for ultraproducts implies that $A^{D} \models \phi$ (this is because in $A_{n}$ the equation $x=a x$ has exactly one solution, namely $a^{n}$, while the equation $y^{2}=a y^{2}$ is satisfied by any of $a^{k}, a^{k+1}, \ldots, a^{n}$, where $\left.k=\lfloor(n-1) / 2\rfloor\right)$. However, since the only solution of the system $\boldsymbol{x}=a x, y^{2}=a y^{2}$ (in $\left.A^{\infty}\right)$ is $\left(a^{\omega}, a^{\omega}\right)$ the sentence $\phi$ cannot be valid in $A^{\infty}$.

It is also possible to define nonstandard processes in the ultraproduct model. Inded, for any function $\sigma: N \rightarrow N$ such that for all $n>0 \sigma(n) \leqslant n$ define the element

$$
a^{\sigma}=\left[a^{\sigma(n)}: n>0\right]_{D}
$$

For such functions $\sigma$ it is possible to show that

Proposition 4.2. $a^{\sigma} \in A^{\infty}$ if and only if $(\exists X \in D)$ [ $\sigma$ is either constant on $X$ or the identity on $X$ ].

Example 4.3. [In the signature $\left.+, \cdot\|\|,, \mid, \partial_{H}, a(a \in A)\right]$ : For different $A$ 's the models $A^{\infty}$ are not necessarily elementarily equivalent. To see this consider two distinct atoms $a, b$ and define the formula $\phi \equiv(\exists x, y)\left[x=x^{2} \wedge y=y^{2} \wedge x \neq y\right]$. Then it is easy to show that $\{a\}^{\infty}=\neg \phi$, while $\{a, b\}^{\infty}=\phi$.

\section{Discussion and Open Problems}

The proof of the approximation principle requires the compactness of the topological space $A^{\infty}$. This not only forces the set $A$ of atoms to be finite, but it also excludes the possibility of using $\tau$ (silent or internal action). It is not known, however, if the approximation principle could be proved for the same class of positive formulas without these restrictions. The ultraproduct construction is quite general and it seems it would be interesting to study the ultraproduct obtained when one takes countably many copies of the finite term model $A_{\omega}$ (which, by the way, is no longer an extension of the projective model), as well as its relation to the so called graph models (see [BK]). It might also be possible to use the ultraproduct construction in order to prove that certain concepts in process algebra are undefinable in a given signature (see [E], corollary 3.4).

\section{REFERENCES}

[AN] Arnold, A. and Nivat, M., The Metric Space of Infinite Trees: Algebraic and Topological Properties, Fundamenta Informatica, 3, 4(1980), pp. 445-476.

[dBZ] de Bakker, J. W. and Zucker, J. I., Denotational Semantics of Concurrency, Proceedings 14th STOC, pp. 153-158, 1982.

[BK] Bergstra, J. A. and Klop, J. W., Algebra of Communicating Processes, in: Proceedings of the CWI Symposium on Mathematics and Computer Science, J. W. de Bakker, M. Hazenwinkel and J. K. Lenstra, eds., 1986.

[CK] Chang, C. C. and Keisler, H. J., Model Theory, North-Holland, 1973.

[Du] Dugundji, J., Topology, Allyn and Bacon, 1966. 
[Di] Dieudonne, J., Elements d' Analyse, Tom 1, Gauthier-Villars, Paris, 1968.

[E] Eklof. P. C., Ultraproducts for Algebraists, in: Handbook of Mathematical Logic, Barwise, J., ed., pp. 105-137, North-Holland, 1977.

[H] Hoare, C. A. R., Communicating Sequential Processes, Prentice/Hall, 1985.

[K] Kranakis, E., Fixed Point Equations with Parameters in the Projective Model, CWI Technical Report, Computer Science/Department of Algorithms and Architectures, Report CS-R8606, January 1986, to appear.

[L] Lloyd, J., Foundations of Logic Programming, Springer Verlag, 1984.

[M] Milner, R., A Calculus of Communicating Systems, Springer Verlag Lecture Notes in Computer Science, Vol. 92, 1980.

[Ro] Rounds, W. C., Applications of Topology to Semantics of Communicating Processes, in: Seminar in Concurrency, Springer Verlag Lecture Notes in Computer Science, Vol. 197, 1985, pp. 360-372. 